\title{
Estimation of Clinical Predictive Factors in Treating Patients With Globus
}

\author{
Jung Hwan Oh
}

Division of Gastroenterology, College of Medicine, The Catholic University of Korea, Seoul, Korea

Article: Clinical predictors for response to proton pump inhibitor treatment in patients with globus Jeon HK, Kim GH, Choi MK, et al

(J Neurogastroenterol Motil 2013;19:47-53)

Gastroesophageal reflux disease (GERD) may cause laryngopharyngeal reflux (LPR), which results in mucosal exposure of the pharynx, larynx or pulmonary system to the different components of the gastric refluxate, and so provokes symptoms including throat discomfort, hoarseness, globus pharyngeus and chronic cough. Of the LPR patients, 25\% experience spontaneous resolution of symptoms and $50 \%$ have a chronic course of the disease with intermittent exacerbations and remissions. ${ }^{1}$ There is no gold standard tool for establishing the association between GERD and extraesophageal symptoms of GERD because many of them may have a variety of etiologies. A recent study demonstrated that $52 \%$ of patients had laryngeal symptoms that were associated with GERD based on upper gastrointestinal endoscopy and/or on esophageal $\mathrm{pH}$ monitoring. ${ }^{2}$

Globus is usually long-lasting and difficult to treat in clinical field. Potential diverse causes can lead to globus. Recent data have focused on GERD, abnormalities of the upper esophageal sphincter, psychological and psychiatric disorders, and stress as major factors contributing to the globus sensation. ${ }^{3}$ Since there is a lack of well-designed controlled studies on the treatment of globus, an empirical therapeutic trial with a proton pump inhibitor (PPI) twice daily for at least 3 months is recommended in patients with a clinically suspected reflux-related extraesophageal symptoms in Westerns. ${ }^{4}$ In this issue of Journal of Neurogastroenterology and Motility, Jeon et al ${ }^{5}$ investigated clinical predictors to short-term PPI treatment in 41 patients with globus treated with pantoprazole $40 \mathrm{mg}$ daily for 4 weeks. Of them, 22 patients (53.7\%) were classified as responders. They suggested that the presence of typical reflux symptoms was related to a higher response rate to 4-week pantoprazole treatment, which means globus is associated with GERD. On the contrary to this study, another study showed that typical reflux symptoms were not associated with improvement of globus symptoms in which patients with globus received rabeprazole $20 \mathrm{mg}$ twice daily for 14 days. ${ }^{6}$ Apart from relation of reflux, stress and psychological factors have often been thought to trigger the globus sensation. A psychological history should be considered and could be an important predictor for treatment outcome, because anxiety is related to poorer response to PPI therapy. ${ }^{7}$ Authors also suggested that long symptom duration ( $\geq 3$ months) was associated with a

Received: December 19, 2012 Revised: December 22, 2012 Accepted: December 24, 2012

(c) This is an Open Access article distributed under the terms of the Creative Commons Attribution Non-Commercial License (http://creativecommons. org/licenses/by-nc/3.0) which permits unrestricted non-commercial use, distribution, and reproduction in any medium, provided the original work is properly cited.

*Correspondence: Jung Hwan Oh, MD

Department of Internal Medicine, The Catholic University of Korea College of Medicine, St. Paul's Hospital, 180, Wangsan-ro, Dongdaemun-gu, Seoul 130-709, Korea

Financial support: None.

Tel: +82-2-958-2343, Fax: +82-2-968-7250, E-mail: ojh@catholic.ac.kr

Conflicts of interest: None. 
lower response rate. It is dubitable whether the effect of good response in patients with short symptom duration would be due to the medication or placebo effect. Further study will be needed only in patients with long symptom duration ( $\geq 3$ months) excluding short duration, which will have much more significant results. This study is not a placebo controlled design, which makes the results difficult to apply in real clinical field. There were previous studies which were conducted as a prospective, randomized, double-blind, placebo-controlled trial. They have shown conflicting results. ${ }^{8,9}$ A study showed that twice-daily PPI treatment for 3 months demonstrated a significantly greater improvement in laryngeal appearance and LPR symptoms, ${ }^{8}$ while another study suggested that the patient with globus did not benefit from once-daily lansoprazole therapy for 3 months. ${ }^{9}$ The difference between two studies is that participants with all other LPR symptoms as well as globus were included in the former study, and in addition, higher dose of PPI were used compared to the latter one.

Inadequate duration of treatment would be an issue, because at least 3 months of treatment has been suggested in patients with LPR in Westerns. There is no guideline in Easterns, and authors explained that 7-14 days is considered to be probably long enough to determine the effect of the PPI. ${ }^{10}$ Limitations of the current study include not a placebo controlled study, its small sample size and without history of psychological factors. Followup data regarding natural course of patients with globus should be needed as well because responding with short-term therapy does not mean symptom free for long-term.

In summary, the cause of globus seems to be multifactorial. The lack of evidence still exists in association with GERD. This study showed globus is connected with GERD and suggested clinical predictors in treating globus symptom with PPI. A guideline to treat LPR patients would be drawn up through much more researches in Eastern.

\section{References}

1. Koufman JA. The otolaryngologic manifestations of gastroesophageal reflux disease (GERD): a clinical investigation of 225 patients using ambulatory 24-hour $\mathrm{pH}$ monitoring and an experimental investigation of the role of acid and pepsin in the development of laryngeal injury. Laryngoscope 1991;101(4 Pt 2 suppl 53):1-78.

2. Oh JH, Choi MG, Park JM, et al. The clinical characteristics of gastroesophageal reflux disease in patients with laryngeal symptoms who are referred to gastroenterology. Dis Esophagus Published Online First: 20 Jul 2012. doi: 10.1111/j.1442-2050.2012.01375.x.

3. Lee BE, Kim GH. Globus pharyngeus: a review of its etiology, diagnosis and treatment. World J Gastroenterol 2012;18:2462-2471.

4. Labenz J. Facts and fantasies in extra-oesophageal symptoms in GORD. Best Pract Res Clin Gastroenterol 2010;24:893-904.

5. Jeon HK, Kim GH, Choi MK, et al. Clinical predictors for response to proton pump inhibitor treatment in patients with globus. J Neurogastroenterol Motil 2013;19:47-53.

6. Sinn DH, Kim JH, Kim S, et al. Response rate and predictors of response in a short-term empirical trial of high-dose rabeprazole in patients with globus. Aliment Pharmacol Ther 2008;27:1275-1281.

7. Heading RC, Mönnikes H, Tholen A, Schmitt H. Prediction of response to PPI therapy and factors influencing treatment outcome in patients with GORD: a prospective pragmatic trial using pantoprazole. BMC Gastroenterol 2011;11:52.

8. Reichel O, Dressel H, Wiederänders K, Issing WJ. Double-blind, placebo-controlled trial with esomeprazole for symptoms and signs associated with laryngopharyngeal reflux. Otolaryngol Head Neck Surg 2008;139:414-420.

9. Dumper J, Mechor B, Chau J, Allegretto M. Lansoprazole in globus pharyngeus: double-blind, randomized, placebo-controlled trial. J Otolaryngol Head Neck Surg 2008;37:657-663.

10. Bruley des Varannes S. The proton-pump inhibitor test: pros and cons. Eur J Gastroenterol Hepatol 2004;16:847-852. 